\title{
Correction to: Exploring the association between whole blood Omega-3 Index, DHA, EPA, DPA, AA and n-6 DPA, and depression and self-esteem in adolescents of lower general secondary education
}

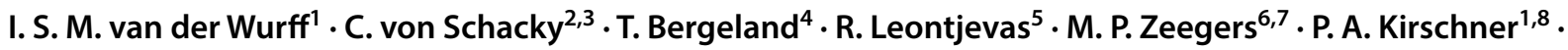 \\ R. H. M. de Groot ${ }^{1,6}$
}

Published online: 10 January 2020

(c) The Author(s) 2020

\section{Correction to: \\ European Journal of Nutrition (2019) 58:1429-1439 https://doi.org/10.1007/s00394-018-1667-4}

The original version of this article unfortunately contained a mistake. Title was incorrect.

The correct title should be:

Exploring the association between whole blood Omega-3 Index, DHA, EPA, DPA, AA and n-6 DPA, and depression and self-esteem in adolescents of lower general secondary education

Open Access This article is distributed under the terms of the Creative Commons Attribution 4.0 International License (http://creativeco

The original article can be found online at https://doi.org/10.1007/ s00394-018-1667-4.

\section{S. M. van der Wurff}

inge.vanderwurff@ou.nl

1 Welten Institute, Research Centre for Learning, Teaching, and Technology, Open University of the Netherlands, Valkenburgerweg 177, P.O. Box 2960, 6419 AT Heerlen, The Netherlands

2 Omegametrix, 82152 Martinsried, Germany

3 Preventive Cardiology, Medical Clinic and Poli-Clinic I, Ludwig Maximilians-University Munich, Munich, Germany

4 Aker BioMarine Antarctic AS, 1327 Lysaker, Norway

5 Faculty of Psychology and Educational Sciences, Open University of the Netherlands, 6419 AT Heerlen, The Netherlands

6 Nutrition and Translational Research in Metabolism (School NUTRIM), Maastricht University, 6200 MD Maastricht, The Netherlands

7 Care and Public Health Research Institute (School CAPHRI), Maastricht University, 6200 MD Maastricht, The Netherlands

8 University of Oulu, Oulu, Finland mmons.org/licenses/by/4.0/), which permits unrestricted use, distribution, and reproduction in any medium, provided you give appropriate credit to the original author(s) and the source, provide a link to the Creative Commons license, and indicate if changes were made. 